\title{
Estudo Comparativo e Classificação de Algoritmos LMS de Passo Variável
}

\author{
José Gil F. Zipf, Orlando J. Tobias e Rui Seara
}

\begin{abstract}
Resumo-Os algoritmos adaptativos de passo variável têm grande importância prática, visto que apresentam um melhor desempenho em relação aos de passo fixo. Neste contexto, a literatura técnica apresenta um grande número de trabalhos com esse objetivo, permanecendo ainda hoje como uma linha de pesquisa bastante ativa. Uma das dificuldades que o leitor enfrenta com os trabalhos desta área é a falta de padronização entre os resultados publicados. Assim, os objetivos deste trabalho de pesquisa são os seguintes: (i) unificar a nomenclatura utilizada e (ii) classificar os algoritmos segundo o princípio de ajuste utilizado para o passo de adaptação. A partir deste trabalho, será possível uma melhor compreensão dos diferentes algoritmos discutidos bem como a concepção de outros com um princípio diferente. $O$ trabalho finaliza com alguns resultados de simulação numérica, considerando o mesmo cenário de comparação de desempenho para os algoritmos discutidos.
\end{abstract}

Palavras-chave-Algoritmos adaptativos de passo variável, Algoritmo LMS, Filtros adaptativos.

Abstract-Adaptive algorithms of variable step size have a large practical importance, since they present a better performance with respect to the ones having fixed step size. In this context, the technical literature presents a large number of works with this aim, remaining today as a very active research area. One of the difficulties that the reader faces with such an amount of results is the lack of standardization between the published materials. Thus, the objectives of this research work include: (i) to unify the used nomenclature, and (ii) to classify the algorithms according to the adjusting principle of the adaptation step size. From this work, a better understanding of these algorithms is expected as well as the conception of others with a different principle may be favored. This work concludes with some results of numerical simulations, considering the same performance comparison environment for the discussed algorithms.

Keywords-Variable step size adaptive algorithms, LMS algorithm, Adaptive filters.

\section{INTRODUÇÃO}

O algoritmo LMS tornou-se o algoritmo mais popular em aplicações de filtragem adaptativa, devido à sua simplicidade e robustez nos mais variados cenários [1]. O algoritmo LMS convencional opera com um passo de adaptação fixo. Nessa condição, existe um compromisso para a escolha do valor do

José Gil F. Zipf e Rui Seara, LINSE - Laboratório de Circuitos e Processamento de Sinais, Depto. de Eng. Elétrica, Universidade Federal de Santa Catarina, Florianópolis, SC, E-mails: \{gil, seara\}@linse.ufsc.br.

Orlando J. Tobias, LINSE/UFSC e Departamento de Engenharia Elétrica e Telecomunicações, Universidade Regional de Blumenau, SC, E-mail: tobias.oj@ieee.org.

Este trabalho foi parcialmente financiado pelo Conselho Nacional de Desenvolvimento Científico e Tecnológico (CNPq). passo de adaptação. Quanto maior for esse valor, desde que ele esteja dentro do limite de estabilidade, maior será, nesse caso, a velocidade de convergência do algoritmo, porém, maior será o erro em regime permanente. Por outro lado, um passo de valor pequeno proporciona valores de desajuste menores em regime permanente ao custo de uma convergência mais lenta.

Com o objetivo de se obter elevadas taxas de convergência com um desajuste reduzido, várias estratégias para ajustar o valor do passo de adaptação dinamicamente vêm sendo propostas na literatura, resultando nos denominados algoritmos LMS de passo variável (VSLMS). As aplicações dos algoritmos de passo variável são basicamente as mesmas dos algoritmos convencionais: identificação de sistemas, cancelamento de ruído [1], cancelamento de eco [1], equalização de canal [1], dentre outras. A idéia básica desses algoritmos é utilizar um valor de passo de adaptação elevado no início do processo de convergência e gradualmente reduzi-lo até se atingir o regime permanente. Para tal, vários autores têm proposto diferentes estratégias para a atualização do passo de adaptação [2]-[17]. Assim, um problema surge decorrente da quantidade de trabalhos nesta área; a grande maioria desses trabalhos não apresenta uma padronização que permita estabelecer comparações de desempenho frente a cenários comuns de aplicação. Nesse contexto, torna-se difícil realizar a escolha de um certo tipo de estratégia de ajuste. Assim, este artigo apresenta as principais estratégias de algoritmos VSLMS, classificando-as em diferentes categorias, segundo a idéia central de cada algoritmo. Um ponto interessante a se destacar é que todos os algoritmos são estudados usando o mesmo cenário, permitindo agora estabelecer uma comparação de desempenho mais confiável.

Em resumo, este artigo contribui particularmente com os seguintes pontos: (i) uma padronização da nomenclatura para os diferentes algoritmos VSLMS; (ii) estabelece uma classificação das diversas estratégias disponíveis na literatura. Dessa forma, este trabalho reúne em uma única fonte de consulta um resumo dos principais algoritmos VSLMS, destacando suas principais características. Alguns resultados de simulação numérica são apresentados, considerando o mesmo cenário de comparação de desempenho para os algoritmos aqui discutidos.

\section{Algoritmo VSLMS}

Esta seção apresenta um equacionamento geral para o algoritmo VSLMS, considerando para tal um esquema de identificação de sistemas, conforme ilustrado na Fig. 1. 


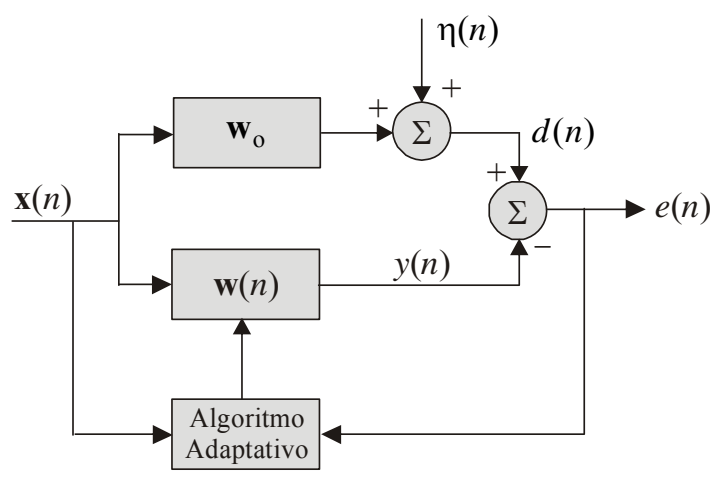

Fig. 1. Diagrama de blocos para identificação de sistemas.

A saída do sistema a ser identificado é dada por

$$
d(n)=\mathbf{w}_{\mathrm{o}}^{\mathrm{T}} \mathbf{x}(n)+\eta(n)
$$

onde $\mathbf{x}(n)$ denota o processo de entrada com média zero e variância $\sigma_{x}^{2}, \eta(n)$ é um ruído de medição com média zero e variância $\sigma_{\eta}^{2}$, descorrelacionado de qualquer outro sinal no sistema. A variável $\mathbf{w}(n)$ representa o vetor de coeficientes de ordem $M$ e $\mathbf{w}_{\mathrm{o}}$, a resposta ao impulso do sistema a ser identificado. O sinal de erro é dado por

$$
\mathrm{e}(n)=d(n)-\mathbf{w}^{\mathrm{T}} \mathbf{x}(n) .
$$

A equação de atualização do vetor de coeficientes, considerando o algoritmo VSLMS, tem a seguinte forma:

$$
\mathbf{w}(n+1)=\mathbf{w}(n)+\mu(n) e(n) \mathbf{x}(n)
$$

onde $\mu(n)$ representa o passo variável de adaptação. Algumas estratégias utilizam um passo individual para cada componente do vetor de coeficientes. Nesse caso, o passo de adaptação $\mu(n)$ é substituído por uma matriz diagonal $\mathbf{D}(n)$, cuja diagonal principal é composta pelos passos individuais de adaptação

$$
\mathbf{D}(n)=\left[\begin{array}{ccccc}
\mu_{0}(n) & 0 & 0 & \ldots & 0 \\
0 & \mu_{1}(n) & 0 & \ldots & 0 \\
0 & 0 & \mu_{2}(n) & \ldots & 0 \\
\vdots & \vdots & \vdots & \ddots & \vdots \\
0 & 0 & 0 & \ldots & \mu_{M-1}(n)
\end{array}\right] .
$$

Neste caso, (3) é expressa como

$$
\mathbf{w}(n+1)=\mathbf{w}(n)+e(n) \mathbf{D}(n) \mathbf{x}(n) .
$$

Um ponto importante nos algoritmos VSLMS é a limitação da variação do passo de adaptação para garantir a estabilidade do algoritmo, devendo satisfazer a seguinte relação de desigualdades [1]:

$$
0<\mu(n)<\frac{2}{3 \operatorname{tr}[\mathbf{R}]}
$$

onde $\mathbf{R}$ é a matriz de autocorrelação do vetor de entrada.

\section{ClassifiCAÇÃo dos Algoritmos VSLMS}

Nesta seção, é realizada a classificação dos algoritmos LMS de passo variável. Para tal, são estabelecidas seis categorias, conforme o critério de ajuste do passo de adaptação, que podem ser baseadas em:

i) Gradiente do erro quadrático.

ii) Erro quadrático.

iii) Autocorrelação do erro.

iv) Valor absoluto do erro.

v) Normalização do vetor de erros.

vi) Ajuste proporcional aos valores absolutos dos elementos do vetor de coeficientes.

Ao todo, são analisados 14 algoritmos VSLMS, sendo discutidos aqueles mais representativos de cada classe, dentre um grande número de algoritmos disponível na literatura. A seguir, são apresentados os algoritmos inseridos em cada uma das categorias supramencionadas.

\section{A. Algoritmos Baseados no Gradiente}

Nesta categoria, estão incluídos os algoritmos VSLMS cujo ajuste do passo de adaptação é realizado de maneira proporcional ao gradiente do valor quadrático do erro de estimação.

Os primeiros trabalhos baseados nesta abordagem [18] baseavam-se apenas nas trocas de sinais, observadas nos componentes do gradiente do erro quadrático a cada iteração. Posteriormente, foram propostos algoritmos que utilizavam o valor numérico do gradiente para efetuar um ajuste proporcional do passo de adaptação. Em todos os casos, utiliza-se uma estimação do gradiente. Assim, a presença de ruído nessa estimativa geralmente leva a um aumento do desajuste do algoritmo. Os principais algoritmos incluídos nessa categoria são:

1) Algoritmo de Shan [6]. Utiliza uma estimativa da correlação entre o sinal de erro de adaptação e o sinal de entrada, determinada através da seguinte expressão:

$$
\nabla(n)=\lambda \nabla(n-1)+(1-\lambda) e(n) \bar{x}(n)
$$

onde $\bar{x}(n)=\frac{1}{M} \sum_{k=n-M+1}^{n} x(k)$, para $\lambda$ considerado um fator de esquecimento. O ajuste do passo de adaptação é então dado por

$$
\mu(n)=\alpha \nabla(n)
$$

onde o fator $\alpha$ é uma constante positiva menor do que um.

2) Algoritmo de Richards [2]. Essa estratégia opera com passos individuais de adaptação, utilizando a potência média do gradiente para calcular o passo de adaptação. Nesse algoritmo, a matriz diagonal de passo de adaptação é obtida a partir da potência da estimativa do gradiente, dada por 


$$
\hat{\nabla}(n)=\left[\begin{array}{c}
\frac{\partial e^{2}(n)}{\partial w_{0}} \\
\vdots \\
\frac{\partial e^{2}(n)}{\partial w_{M-1}}
\end{array}\right]=-2 e(n) \mathbf{x}(n) .
$$

Para se obter o valor médio do gradiente, é aplicado um filtro passa-baixas de média móvel, dado por

$$
E\left[\nabla_{i}(n+1)\right]=\beta E\left[\nabla_{i}(n)\right]+(1-\beta) e(n) x(n-i)
$$

onde $\nabla_{i}(n)$ representa o $i$-ésimo elemento do vetor gradiente e $\beta$ é um parâmetro que controla a seletividade do filtro.

Então, a matriz $\mathbf{D}(n)$ é dada por

$$
\mathbf{D}(n)=\gamma\left[\begin{array}{cccc}
E\left[\nabla_{0}(n)\right] & 0 & \cdots & 0 \\
0 & E\left[\nabla_{1}(n)\right] & \cdots & 0 \\
\vdots & \vdots & \ddots & \vdots \\
0 & 0 & \cdots & E\left[\nabla_{M-1}(n)\right]
\end{array}\right]
$$

onde $\gamma$ é um parâmetro positivo de controle do passo de adaptação.

3) Algoritmo de Mathews [3]. A idéia central desse algoritmo é ajustar o passo de adaptação de forma proporcional ao gradiente do erro quadrático em relação aos passos anteriores. A equação de ajuste é

$$
\mu(n)=\mu(n-1)-\frac{\rho}{2} \frac{\partial e^{2}(n)}{\partial \mu(n-1)} .
$$

A expressão (12) pode ser escrita da seguinte forma:

$$
\mu(n)=\mu(n-1)-\frac{\rho}{2} \frac{\partial^{\mathrm{T}} e^{2}(n)}{\partial \mathbf{w}(n)} \frac{\partial \mathbf{w}(n)}{\partial \mu(n-1)}
$$

após a determinação das derivadas parciais, resulta em

$$
\mu(n)=\mu(n-1)+\rho e(n) e(n-1) \mathbf{x}^{\mathrm{T}}(n-1) \mathbf{x}(n)
$$

onde $\rho$ é um parâmetro positivo de controle de passo. $\mathrm{Na}$ abordagem de Mathews, é também possível utilizar passos individuais para cada coeficiente do filtro, resultando na seguinte equação de ajuste:

$$
\mu_{i}(n)=\mu_{i}(n-1)+\rho e(n) e(n-1) x_{i}(n) x_{i}(n-1), \quad i=0, \cdots, M-1 .
$$

4) Algoritmo de Wee-Peng Ang [8]. Esse algoritmo utiliza uma forma recursiva para determinação do termo $\frac{\partial \mathbf{w}(n)}{\partial \mu(n-1)}$ do algoritmo de Mathews (13), através de um filtro de média móvel, dado por

$$
\psi_{i}(n)=\frac{\partial w_{i}(n)}{\partial \mu_{i}(n-1)}=\alpha \psi_{i}(n-1)+x_{i}(n-1) e(n-1)
$$

onde $\alpha$ é um parâmetro de controle de seletividade do filtro. Assim, a partir de (2), a equação de ajuste do passo de adaptação é dada por

$$
\mu_{i}(n)=\mu_{i}(n-1)+\rho e(n) x_{i}(n) \psi_{i}(n)
$$

onde $\rho$ é o parâmetro de ajuste do passo de adaptação do algoritmo VSLMS em questão.

Note que o algoritmo de Mathews é obtido fazendo $\alpha=0$ em (16). Para o caso de um único passo de adaptação, as equações utilizadas são

$$
\psi(n)=\alpha \psi(n-1)+e(n-1) \mathbf{x}(n-1)
$$

$$
\mu(n)=\mu(n-1)+\rho e(n) \mathbf{x}^{\mathrm{T}}(n) \psi(n)
$$

onde $\alpha$ e $\rho$ são parâmetros de controle de adaptação.

5) Algoritmo de Farhang [5]. Esse algoritmo é obtido a partir de modificações no algoritmo de Mathews. Em [5], é proposto, para o caso de múltiplos passos de adaptação, a seguinte expressão:

$$
\mu_{i}(n+1)=\left[1+\delta_{i} e(n) x_{i}(n) e(n-1) x_{i}(n-1)\right] \mu_{i}(n)
$$

onde o parâmetro de controle $\delta_{i}$ é obtido através de

$$
\delta_{i}=\frac{\delta_{o}}{\varepsilon_{i}} .
$$

Em (21), $\delta_{o}$ é uma constante fixa e $\varepsilon_{i}$ representa o valor médio de $\left|e(n) x_{i}(n)\right|^{2}$.

6) Algoritmo de Okello [7]. O ajuste do passo variável é baseado na potência média da estimativa do gradiente do erro quadrático, dada por

$$
\mu(n)=\alpha \mu(n-1)+\sigma \sum_{i=0}^{M-1} p_{i}^{2}(n)
$$

onde

$$
p_{i}(n)=\beta p_{i}(n-1)+e(n) x_{i}(n)
$$

é a estimativa do gradiente do erro quadrático em relação ao i-ésimo componente do vetor de coeficientes, e $\alpha$ e $\beta$ são os parâmetros de controle de adaptação.

\section{B. Algoritmos Baseados no Erro Quadrático}

Nesta categoria, os algoritmos ajustam o passo utilizando o valor do erro quadrático instantâneo. Pela natureza de ajuste do passo, esses algoritmos possuem, em geral, uma forte dependência com o ruído de medição. Assim, as diferentes estratégias propostas para essa classe de algoritmos buscam minimizar tal dependência. Em geral, o desempenho desse tipo de algoritmo é afetado por ambientes com baixa razão sinal-ruído.

1) Algoritmo de Kwong [9]. Nesse algoritmo, o ajuste do passo de adaptação é controlado pelo quadrado do erro de predição, conforme a seguinte expressão:

$$
\mu(n+1)=\alpha \mu(n)+\gamma e^{2}(n)
$$

onde $\alpha$ e $\gamma$ são parâmetros de controle de ajuste do passo.

A idéia por trás deste algoritmo é que um erro de predição grande leva a um aumento no valor do passo, produzindo, assim, uma convergência mais rápida. Por outro lado, um 
valor reduzido de erro resulta em um valor de passo menor e, conseqüentemente, em um baixo desajuste.

2) Algoritmo de Nakanishi [10]. O ajuste do passo de adaptação desse algoritmo é baseado no erro quadrático instantâneo e na norma $l_{\infty}$ do vetor de erro quadrático, segundo a expressão

$$
\mu(n)=\mu\left[1-\frac{e^{2}(n)_{\max }}{\alpha e^{2}(n)}\right]
$$

onde $e^{2}(n)_{\max }$ é o maior valor de erro quadrático entre a primeira e a $n$-ésima iteração, e $\alpha$ e $\mu$ são parâmetros de ajuste do passo de adaptação.

3) Algoritmo de Costa [11]. Determinado a partir de uma modificação no algoritmo de Kwong, visando torná-lo menos susceptível ao ruído de medição, esse algoritmo é baseado na seguinte equação de ajuste:

$$
\mu(n)=\alpha \mu(n-1)+\gamma\left[k \mathbf{x}^{\mathrm{T}}(n) \mathbf{x}(n)-1\right] e^{2}(n), \quad k=\frac{1}{M \sigma_{\mathrm{x}}^{2}}
$$

onde $\alpha$ e $\gamma$ são parâmetros de ajuste do passo de adaptação. A inserção do fator $\left[k \mathbf{x}^{\mathrm{T}}(n) \mathbf{x}(n)-1\right]$ em (26) tem por objetivo reduzir a influência do ruído de medição no ajuste do passo de adaptação.

\section{Algoritmos Baseados na Autocorrelação do Erro}

Para esta classe de algoritmos, o passo é ajustado com base na autocorrelação entre $e(n)$ e $e(n-1)$. Com isso, o algoritmo pode efetivamente ajustar o valor do passo, mantendo uma aceitável imunidade a ruídos de medição não correlacionados.

1) Algoritmo de Aboulnasr [12]. Nesse algoritmo, o passo de adaptação é ajustado considerando o quadrado da estimativa da autocorrelação entre $e(n)$ e $e(n-1)$, obtida através de um filtro de média móvel, dado por

$$
p(n)=\beta p(n-1)+(1-\beta) e(n) e(n-1)
$$

onde $\beta$ é um parâmetro que controla a seletividade do filtro. A equação proposta para realizar o ajuste do passo de adaptação é

$$
\mu(n+1)=\alpha \mu(n)+\gamma p^{2}(n)
$$

onde $\alpha$ e $\gamma$ são parâmetros de ajuste do passo de adaptação. A utilização de $p(n)$ na atualização de $\mu(n)$ atende a dois objetivos: a função de autocorrelação do erro é, em geral, uma adequada medida da proximidade do ponto ótimo e, em segundo lugar, fornece uma certa imunidade a ruídos de medição não correlacionado.

\section{Algoritmos Baseados no Valor Absoluto do Erro}

Nesta categoria, o ajuste do passo variável do algoritmo VSLMS é realizado com base no módulo do erro instantâneo de adaptação.
1) Algoritmo de Kim [13]. Nesse algoritmo, o passo é determinado com base no valor absoluto do erro de estimação. A equação de ajuste do passo proposta tem a seguinte forma:

$$
\mu(n)=\frac{|e(n)|}{|d(n)|} \mu_{\max }
$$

$\mathrm{e}$

$$
\mu(n)= \begin{cases}\alpha \mu(n-1), & \mu(n)<\alpha \mu(n-1) \\ \frac{1}{\alpha} \mu(n-1), & \mu(n)>\frac{1}{\alpha} \mu(n-1) \\ \mu(n), & \text { caso contrário }\end{cases}
$$

onde $0<\alpha<1$ é o fator de controle de convergência do passo de adaptação.

\section{E. Algoritmos Baseados na Normalização do Vetor de Erros}

Nesta categoria, a atualização do passo é realizada com base na norma do vetor de erro de estimação.

1) Algoritmo de Ramadan [14]. Nesse algoritmo, a estratégia de atualização do passo depende de uma regularização, baseada em uma função não-linear, das normas dos vetores de erros e do sinal de entrada do filtro adaptativo. A partir da recursão de Newton e utilizando aproximações instantâneas para $\mathbf{R}=\mathrm{E}\left\{\mathbf{x}(n)^{\mathrm{T}} \mathbf{x}(n)\right\}$ e $\mathbf{p}=\mathrm{E}\{d(n) \mathbf{x}(n)\}$, é proposta a seguinte equação de ajuste:

$$
\mu(n)=\mu\left\|\mathbf{e}_{L}(n)\right\|^{2}\left[\alpha\|\mathbf{e}(n)\|^{2} \mathbf{I}+\gamma \mathbf{x}(n) \mathbf{x}^{\mathrm{T}}(n)\right]^{-1}
$$

onde $\mu, \alpha$ e $\gamma$ são parâmetros constantes positivos.

As normas dos vetores de erro são determinadas a partir de

$$
\|\mathbf{e}(n)\|^{2}=\sum_{k=0}^{n-1}|e(n-k)|^{2}
$$

$\mathrm{e}$

$$
\left\|\mathbf{e}_{L}(n)\right\|^{2}=\sum_{k=0}^{L-1}|e(n-k)|^{2}
$$

Expandindo (31) e usando o lema de inversão de matrizes, obtém-se

$$
\mu(n)=\frac{\mu\left\|\mathbf{e}_{L}(n)\right\|^{2}}{\left[\alpha\|\mathbf{e}(n)\|^{2}+(1-\alpha)\|\mathbf{x}(n)\|^{2}\right]} .
$$

Sem perda de generalidade, a constante $\gamma$ é substituída por $(1-\alpha)$. Os parâmetros $\alpha, L$ e $\mu$ devem ser escolhidos de maneira a se obter o melhor compromisso entre velocidade e desajuste. Uma constante de valor pequeno $\varepsilon$ poderia ser adicionada ao denominador em (34), prevenindo a instabilidade do algoritmo caso o denominador assuma valores muito pequenos. A quantidade $\left\|\mathbf{e}_{L}(n)\right\|^{2}$ é de valor elevado no início da adaptação, decrescendo rapidamente com ao longo das iterações. Por outro lado, $\|\mathbf{x}(n)\|^{2}$ varia em torno do valor $M \sigma_{x}^{2}$. O termo $\|\mathbf{e}(n)\|^{2}$ é uma função 
crescente com o decorrer das iterações, uma vez que e( $n)$ tem dimensão crescente ao longo do processo.

\section{F) Algoritmos Baseados no Ajuste Proporcional aos Valores Absolutos do Vetor $\mathbf{w}$}

Nesta categoria, estão os algoritmos onde o ajuste do passo é proporcional ao valor absoluto de cada componente do vetor de coeficientes $\mathbf{w}$. A idéia central aqui é que, quanto maiores forem os valores dos componentes da resposta ao impulso do sistema, maior deverá ser o valor do correspondente passo de adaptação.

1) Algoritmo de Rohani [15]. A equação de atualização dos passos individuais proposta por esse algoritmo tem a seguinte forma:

$$
\mu_{i}(n)=\beta\left|w_{i}(n)\right|+\mu
$$

onde $\beta$ e $\mu$ são constantes positivas. $\mathrm{O}$ parâmetro $\mu$ é usado como valor inicial para o processo de ajuste, uma vez que todos os coeficientes do vetor $\mathbf{w}$ são normalmente inicializados com o valor zero.

2) Algoritmo de Benesty (IPNLMS) [17]. O algoritmo LMS normalizado proporcional (PNLMS) é apresentado em [16]. $\mathrm{Na}$ adaptação do PNLMS, o ajuste do passo de adaptação é rigorosamente proporcional ao valor do coeficiente. Entretanto, esse algoritmo apresenta um desempenho pior do que o NLMS quando a resposta ao impulso do sistema desconhecido é do tipo dispersiva [17]. Para resolver tal deficiência, em [17] é proposta uma modificação, originando o algoritmo PNLMS melhorado (IPNLMS), baseado na mesma estratégia de atualização do passo do PNLMS. Assim, tem-se

$$
k_{i}(n)=(1-\alpha) \frac{\|\mathbf{w}(n)\|_{1}}{M}+(1+\alpha)\left|w_{i}(n)\right|
$$

$\mathrm{e}$

$$
\|\mathbf{w}(n)\|_{1}=\sum_{i=0}^{M-1}\left|w_{i}(n)\right| .
$$

O ajuste do passo de adaptação é realizado através das seguintes expressões:

$$
\mu_{i}(n)=\frac{k_{i}(n)}{\|\mathbf{k}(n)\|_{1}}
$$

com

$$
\|\mathbf{k}(n)\|_{1}=\sum_{i=0}^{M-1}\left|k_{i}(n)\right|
$$

\section{Resultados de Simulação}

Nesta seção, são apresentadas simulações numéricas, considerando um problema de identificação de sistemas, visando uma comparação de desempenho dos algoritmos VSLMS aqui discutidos. A planta utilizada é obtida a partir de uma janela de Hanning com 9 coeficientes, dada pelo

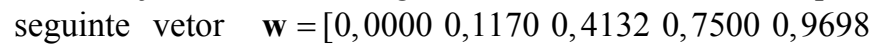

$0,75000,41320,11700,0000]^{\mathrm{T}}$. O sinal de entrada é colorido, obtido a partir de um processo $\operatorname{AR}(1)$, dado por $x(n)=a x(n-1)+u(n)$, considerando $a=0,4$ e $u(n)$ como sendo um ruído branco Gaussiano com $\sigma_{x}^{2}=1$. A dispersão dos autovalores da matriz de autocorrelação do sinal de entrada é $\chi=5$. A variância do ruído aditivo é $\sigma_{\eta}^{2}=0,01(\mathrm{SNR}=20 \mathrm{~dB})$. Para avaliar a capacidade de ajuste (desempenho) dos diversos algoritmos, na iteração $n=300$ a planta é mudada para $-\mathbf{w}_{\mathrm{o}}$, alterando assim o sinal de todos os seus coeficientes. Em todas as simulações, os algoritmos são adequados para se obter o mesmo desajuste em regime permanente ( $10 \%$ do erro mínimo). O algoritmo de Rohani [15] é o único para o qual não foi possível tal ajuste, razão pela qual sua curva de aprendizagem não é mostrada nas figuras apresentadas. As Figs. 2, 3, 4 e 5 mostram os resultados obtidos por simulação Monte Carlo (média de 200 rodadas independentes). A Fig. 2 mostra o comportamento da curva de aprendizagem dos algoritmos da categoria baseada no gradiente. Na Fig. 3, são apresentadas as curvas correspondentes aos algoritmos da classe de erro quadrático e, finalmente, a Fig. 4 apresenta o comportamento dos algoritmos das demais categorias. A Fig. 5 mostra uma comparação entre os algoritmos das Figs. 2, 3 e 4 que apresentaram melhor desempenho.

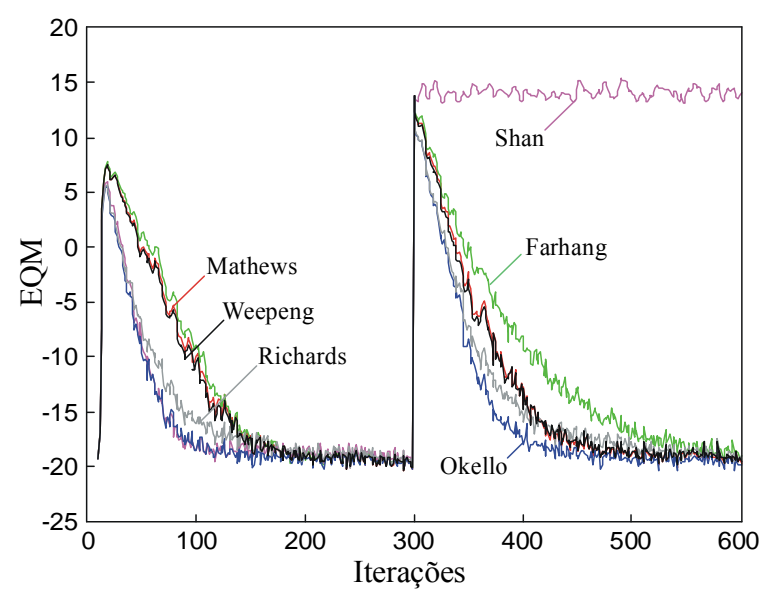

Fig. 2. Algoritmos da categoria baseada no gradiente.

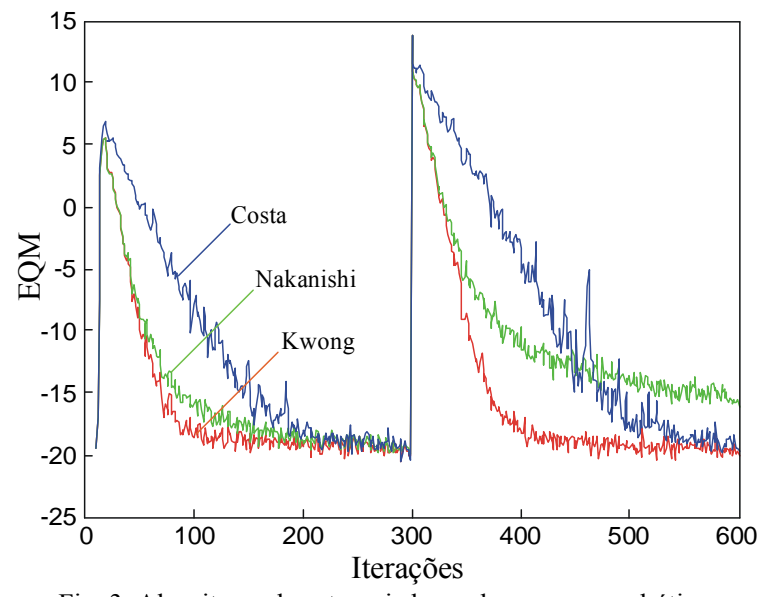

Fig. 3. Algoritmos da categoria baseada no erro quadrático. 


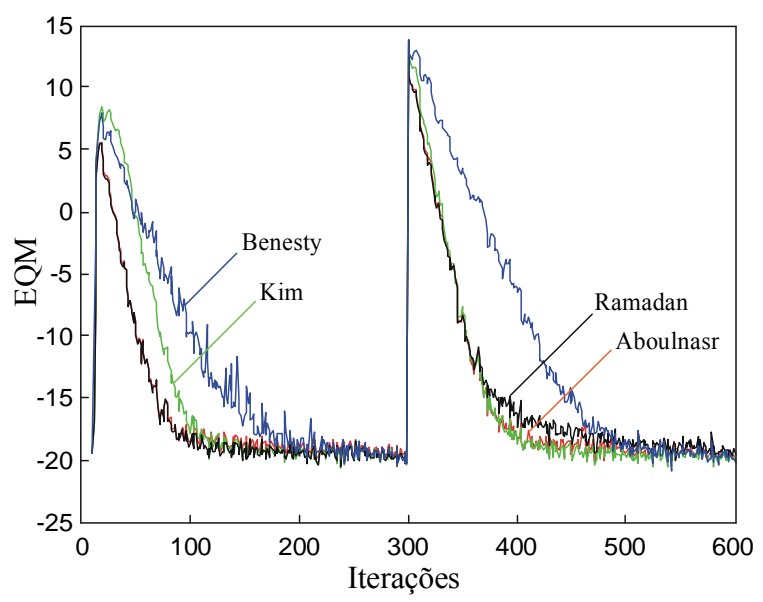

Fig. 4. Algoritmos das demais categorias.

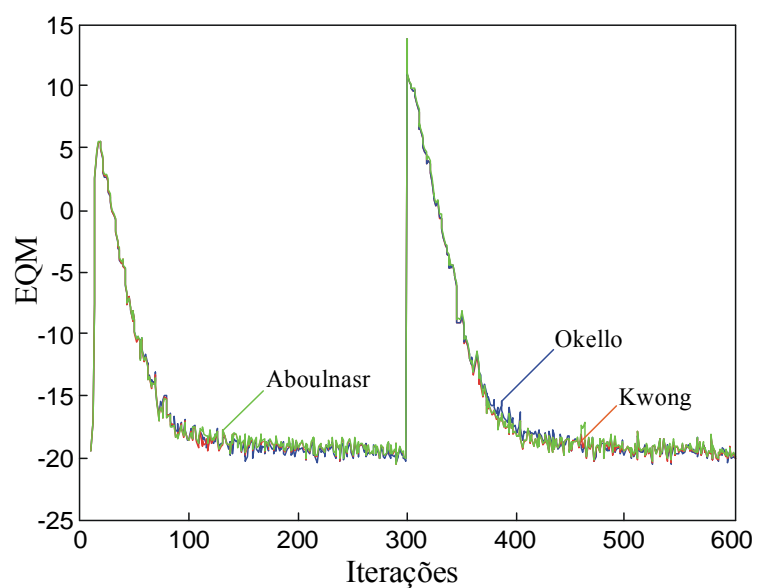

Fig. 5. Algoritmos com melhor desempenho.

Da Fig. 1, destaca-se um desempenho superior do algoritmo de Okello [7]. Também, se observa que, apesar de um bom desempenho na primeira etapa da curva, o algoritmo de Shan [6] não convergiu após a alteração da planta. Da Fig. 2, constata-se um melhor desempenho do algoritmo de Kwong [9] em todas as simulações realizadas; por outro lado, o algoritmo de Nakanishi [10] apresentou um elevado tempo de recuperação, quando da alteração da planta. O desempenho dos algoritmos das demais categorias é apresentado na Fig. 3, na qual verifica-se uma superioridade de desempenho a favor do algoritmo de Aboulnasr [12], baseado na autocorrelação do erro. Finalmente, da Fig. 4, percebe-se que os três algoritmos que apresentaram melhor desempenho possuem suas curvas de aprendizagem bastante semelhantes para a aplicação estudada.

\section{CONCLUSÕES}

Neste trabalho, são estabelecidas bases que permitem realizar uma escolha adequada de um algoritmo adaptativo de passo variável (VSLMS). Para tal, essa classe de algoritmo passou por duas fases, unificação de nomenclatura utilizada e classificação, segundo o princípio de ajuste utilizado para o passo de adaptação. A partir do trabalho proposto, é possível se obter uma melhor compreensão destes algoritmos, bem como o projeto de novos esquemas usando um princípio diferente. Simulações numéricas são apresentadas, comparando o desempenho dos algoritmos aqui discutidos.

\section{REFERÊNCIAS}

[1] S. Haykin, Adaptive Filter Theory, $4^{\text {th }}$ ed., Upper Saddle River, NJ: Prentice Hall, 2002.

[2] J. C. Richards, M. A. Webster, and J. C. Principe, “A gradient-based variable step-size LMS algorithm," in Proc. IEEE Southeastcon, Williamsburg, USA, vol. 2, Apr. 1991, pp. 1083-1087.

[3] V. J. Mathews and Z. Xie, "A stochastic gradient adaptive filter with gradient adaptive step size," IEEE Trans. Signal Process., vol. 41, no. 6, pp. 2075-2087, June 1993.

[4] A. I. Sulyman and A. Zerguine, "Convergence and steady state analysis of a variable step-size normalized LMS algorithm," Proc. IEEE Int. Symp. Signal Processing and Its Applications (ISSPA), Paris, France, vol. 2, July 2003, pp. 591-594.

[5] B. Farhang-Boroujeny, "Variable step size LMS algorithm - New developments and experiments," IEE Proceedings - Vision, Image, Signal Process., vol. 141, no. 5, pp. 311-317, Oct. 1994.

[6] T. J. Shan and T. Kailath, "Adaptive algorithms with an automatic gain control feature," IEEE Trans. Circuits Syst., vol. CAS-35, no. 1, pp. 122-127, Jan. 1988.

[7] J. Okello, Y. Itoh, Y. Fukui, I. Nakanishi, and M. Kobayashi, “A new modified variable step size for the LMS algorithm," in Proc. IEEE Int. Symp. Circuits and Systems (ISCAS), Monterey, USA, vol. 5, Jun. 1998, pp. 170-173.

[8] W. P. Ang and B. Farhang-Boroujeny, "A new class of gradient adaptive step-size LMS algorithms," IEEE Trans. Signal Process., vol. 49, no. 4, pp. 805-810, Apr. 2001.

[9] R. H. Kwong and E. W. Johnston, "A variable step size LMS algorithm," IEEE Trans. Signal Process., vol. 40, no. 7, pp. 1633-1642, July 1992.

[10] I. Nakanishi and Y. Fukui, "A new adaptive convergence factor algorithm with the constant damping parameter," IEICE Trans. Fundamentals, vol. E78-A, no. 6, pp. 649-655, Jun. 1995.

[11] M. H. Costa and J. C. M. Bermudez, "A robust variable step size algorithm for LMS adaptive filters," in Proc. IEEE Int. Conf. Acoust., Speech, Signal Process., Toulouse, France, vol. 3, May 2006, pp. 93-96.

[12] T. Aboulnasr and K. Mayyas, "A robust variable step-size LMS-type algorithm: analysis and simulations", IEEE Trans. Signal Process., vol. 45, no. 3, pp. 631-639, Mar. 1997.

[13] D. W. Kim, J. H. Hoi, Y. S. Choi, C. H. Jeon, and H. Y. Ko, “A VS-LMS algorithm using normalized absolute estimation error", in Proc. IEEE Digital Signal Processing Applications (TENCON), Perth, Australia, vol. 2, Nov. 1996, pp. 692-697.

[14] Z. Ramadan and A. Poularikas, "A robust variable step-size LMS algorithm using error-data normalization," in Proc. IEEE Southeastcon, Huntsville, USA, Apr. 2005, pp. 219-224.

[15] B. Rohani and K. S. Chung, "A modified LMS algorithm with improved convergence," in Proc. IEEE Singapore Int. Conf. Communication Systems, Singapore, Nov. 1994, pp. 845-849.

[16] D. L. Duttweiler, "Proportionate normalized LMS adaptation in echo cancellers", IEEE Trans. Speech Audio Process., vol. 8, no. 5, pp. 508-518, Sept. 2000.

[17] J. Benesty and S. L. Gay, "An improved PNLMS algorithm," in Proc. IEEE Int. Conf. Acoust., Speech, Signal Process., Orlando, USA, May 2002, pp. 1881-1884.

[18] R. W. Harris, D. M. Chabries, and F. A. Bishop, “A variable step (VS) adaptive filter," IEEE Trans. Acoust., Speech, Signal Process., vol. ASSP-34, no. 2, pp. 309-316, Apr. 1986. 